\title{
Leitthema
}

Bundesgesundheitsbl 2019 $62: 186-194$ https://doi.org/10.1007/s00103-018-2869-x Online publiziert: 7. Januar 2019

(c) Der/die Autor(en) 2019

CrossMark
Eva Rothermund ${ }^{1,2} \cdot$ Reinhold Kilian ${ }^{3} \cdot$ Elisabeth M. Balint ${ }^{1,2} \cdot$ Edit Rottler' $^{1}$ Jörn von Wietersheim ${ }^{1} \cdot$ Harald Gündel ${ }^{1,2} \cdot$ Michael Hölzer $^{2,4}$

' Klinik für Psychosomatische Medizin und Psychotherapie, Universitätsklinikum Ulm, Ulm, Deutschland ${ }^{2}$ Kompetenzzentrum für Seelische Gesundheit am Arbeitsplatz Ulm (LPCU), Universität Ulm, Ulm, Deutschland

${ }^{3}$ Klinik für Psychiatrie und Psychotherapie II, Sektion: Gesundheitsökonomie und Versorgungsforschung am Bezirkskrankenhaus Günzburg, Universität UIm, Ulm, Deutschland

${ }^{4}$ ZfP Südwürttemberg, Sonnenbergklinik, Stuttgart, Deutschland

\section{Wie bewerten Nutzer das neue Versorgungsmodell „Die Psychosomatische Sprechstunde im Betrieb"?}

\section{Ergebnisse einer kontrollierten Beobachtungsstudie}

\section{Einleitung}

Psychische und psychosomatische Erkrankungen ${ }^{1,2}$ kommen mit einer 12 Monats-Prävalenz von rund $20-30 \%$ bei der Bevölkerung in Deutschland häufig vor [1]. Arbeitsbedingungen können die psychische Gesundheit erhalten, jedoch auch psychische Erkrankungen auslösen, mitbedingen oder verstärken [2-4]. Nur etwa $20 \%$ der Betroffenen befinden sich in adäquater Behandlung [5]. Dies mag vertretbar erscheinen, da Untersuchungen postulieren, dass bis zu $50 \%$ der Fälle spontan remittieren $[6,7]$. Andererseits gibt es Belege, dass Früherkennung und Frühintervention bei psychischen Erkrankungen die Prognose positiv beeinflussen [8-11]. Auch ist bekannt, dass die Hürden für eine Inanspruchnahme von Leistungen bei psychischen Erkrankungen vielfältig sind. Dies sind zum

\footnotetext{
1 Im Folgenden der Übersichtlichkeit halber „psychische Erkrankungen“ genannt.

2 Im Sinne von "common mental disorders" in Abgrenzung zu "severe mental disorders" werden in der Regel überwiegend Depression ohne psychotische Symptome, Angsterkrankungen, somatoforme Störungen und Anpassungsstörungen eingeordnet.
}

Beispiel die Angst vor Stigmatisierung, Scham, eingeschränktes Wissen über die Entstehung und Behandlung psychischer Erkrankungen $[12,13]$ und schlechte Erreichbarkeit oder erschwerter Zugang zu passenden Behandlungsangeboten [14].

Der Arbeitsplatz gewinnt in der Früherkennung und Frühbehandlung an Bedeutung [15-18], da Kennzeichen seelischer Belastungen und unspezifische chronische Stressbeschwerden hier in einem frühen Stadium auffällig werden können [19].

Die „Psychosomatische Sprechstunde im Betrieb"(Psychosomatic Consultation in the Workplace, PSIW) entspricht einem psychosomatischen Erstkontakt der ambulanten Regelversorgung (Psychosomatic Outpatient Consultation, PSOC), der konsiliarisch, d.h. aufsuchend, im Betrieb stattfindet. Die Versorgungsleistung PSIW ist ein betrieblich initiiertes Angebot. Sie richtet sich an Beschäftigte mit einer drohenden, beginnenden oder manifesten psychischen Erkrankung und beinhaltet eine individuelle Kooperation zwischen dem Betrieb und einem externen Experten für seelische Gesundheit. Die aktuellen Modelle bilden sich noch nicht in der Sozialgesetzgebung ab. Finanziert werden sie bisher und auch bei den in der vorliegenden Untersuchung teilnehmenden Betrieben durch die Betriebe selbst oder eine Betriebskrankenkasse.

Inhaltlich basiert die „Psychosomatische Sprechstunde im Betrieb“ [20] größtenteils auf dem von Gill 1954 erstmals als ,initial psychiatric interview“ oder im Deutschen als „Erstinterview“ [21] definierten Konzept und verfügt über 4 zentrale Elemente (siehe $\bullet$ Infobox 1 ).

Die diagnostische Einschätzung (B) erfolgt basierend auf der Diagnose, der psychosozialen Situation des Patienten sowie der funktionalen Beeinträchtigungen mit dem Fokus auf der Beziehung zwischen Mensch und Arbeitsumfeld. Die Befähigung zum Selbstmanage-

Infobox 1 Zentrale therapeutische Elemente der "Psychosomatischen Sprechstunde im Betrieb"

A. Herstellen einer Beziehung zwischen Beschäftigtem und Behandler

B. Diagnostische Einschätzung

C. Befähigung des Patienten zum Selbstmanagement

D. Motivation und Unterstützung für weitere Schritte 
ment (C) in der PSIW geht über die patientengerechte Aufklärung sowie die im diagnostischen Assessment gewonnene Einschätzung hinaus. Die Erarbeitung eines ersten psychosomatischen Erklärungsmodells erfolgt unter dosiertem Einbezug von Modellen zu Arbeitsstress, zu organisationalen Konzepten sowie zur psychosomatischen Symptombildung. Wissensvermittlung zu organisationalen Konzepten beinhaltet z.B. den Einfluss von Führungskräfteverhalten auf das Arbeitsklima, den erlebten Stress bzw. den Zusammenhang von Arbeitsunsicherheit und stressassoziierten Erkrankungen. Entsprechend der gewonnenen Einschätzung bestehen die Aufgaben in Punkt (D) darin, bei gegebener Indikation Behandlungsmöglichkeiten $\mathrm{zu}$ skizzieren und den Patienten in einem partizipativen Prozess (C) zu befähigen, eine Motivation für eine ggf. notwendige Behandlung zu entwickeln sowie bei der Behandlungsaufnahme unterstützend zur Seite zu stehen. Der Unterschied der betrieblichen (PSIW) zur in der Regelversorgung stattfindenden Sprechstunde (PSOC) ist die Nähe zum Arbeitsplatz und den etablierten Schnittstellen mit und über den Betriebsarzt bzw. den Sozialberater im Betrieb [22].

Betriebsnahe Versorgungsnetzwerke sind an verschiedenen Orten in Deutschland entstanden, werden jedoch kaum systematisch publiziert und sind wissenschaftlich wenig überprüft [20]. Das beschriebene Modell ist ebenso effektiv wie die Regelversorgung unter Routinebedingungen [23]. Dabei erreicht es Betroffene früher im Erkrankungsverlauf $[16,17]$. Eine Integration der Nutzersicht gibt es nach Wissen der Autoren weder für dieses Angebot noch für vergleichbare Modelle. Bei dem Nutzer im Betrieb bestehen jedoch, anders als bei einem Nutzer von Leistungen der Regelversorgung, besondere Rahmenbedingungen in Bezug auf Verantwortlichkeit und Verbindlichkeiten dem Betrieb gegenüber [24]. Kritisch ist, wenn durch eine Fokussierung auf den individuellen Gesundheitszustand die Notwendigkeit einer menschengerechten Gestaltung der Arbeitsbedingungen aus dem Blick verloren wird [25].
Ein erster wichtiger Schritt, das Angebot PSIW in einem angemessen kritischen Rahmen zu beurteilen, ist deshalb, die Nutzer- und Patientensicht zu untersuchen. Das Konstrukt der Patientenoder Nutzerzufriedenheit hat sich in der Versorgungsforschung zur Überprüfung der Qualität und als Maß für das Behandlungsergebnis etabliert [26-29]. Im vorliegenden Beitrag wird deshalb die Frage untersucht:

Wie zufrieden sind Nutzer des neuen PSIW-Versorgungsmodells im Vergleich zu Nutzern einer etablierten ambulanten Regelversorgung (PSOC) unter Berücksichtigung des Behandlungsergebnisses, kontrolliert für soziodemografische Merkmale, und die Erkrankungsschwere?

\section{Methoden}

\section{Studiendesign und Teilnehmer}

Die vorgestellten Daten sind Teil einer kontrollierten prospektiven Beobachtungsstudie mit dem Titel „Die Psychosomatische Sprechstunde im Betrieb ein neues Versorgungsmodell an der Schnittstelle zwischen betrieblicher Versorgung und Konsiliarpsychosomatik“. Diese Studie umfasst 367 Nutzer mit einem ambulanten psychotherapeutischen Erstkontakt und wird ausführlich im Studienprotokoll beschrieben [30]. Verglichen wurden 174 Angestellte aus drei Unternehmen, die eine „Psychosomatische Sprechstunde im Betrieb“" (PSIW) nutzten, mit 193 Nutzern psychotherapeutischer Ambulanzen der regionalen Regelversorgung (PSOC; [23]). Alle Teilnehmer erhielten 1 bis 2 Sitzungen à 50-60 min. Die Daten wurden mittels Selbstbeurteilungsinstrumente vor Erstkontakt (t1) und 12 Wochen danach (t2) erhoben. Die Bewertung von Angebot/Zufriedenheit sowie die Angabe, ob weitere Therapie stattgefunden hatte, wurden nur zu $\mathrm{t} 2$ erhoben.

Eingeschlossen wurden Teilnehmer ab 18 Jahren mit ausreichenden Deutschkenntnissen, die zum Zeitpunkt der Erhebung in einem Beschäftigungsverhältnis standen. Um an der PSIW teilnehmen zu können, mussten die Nutzer Beschäftigte eines von drei Unternehmen mit die- sem Angebot sein. Je nach Betrieb und dort bestehender Struktur wurden die Mitarbeiter über betriebsinterne Kommunikationskanäle, wie z. B. Intranet, Flyer oder Betriebsversammlung, über das Angebot informiert. Ebenfalls von der jeweiligen Struktur mitbestimmt erfolgte die Zuweisung über den betriebsärztlichen Dienst, eine Sozialberatung und/oder mittels Selbstzuweisung der Mitarbeiter [31]. Die an der Studie teilnehmenden Unternehmen sind international tätige Großunternehmen in den Branchen Metallverarbeitung und Sicherheitstechnik. Die teilnehmenden Standorte beschäftigten jeweils mehr als 3000 Mitarbeiter sowohl in Produktion als auch in der Entwicklung.

Die Teilnehmer der betrieblichen Sprechstunden wurden konsekutiv von November 2011 bis Juni 2013 rekrutiert. Die Nutzer der Sprechstunde in der psychosomatischen Regelversorgung wurden von Juni 2012 bis Januar 2013 konsekutiv in der Hochschulambulanz der Psychosomatischen Klinik der Universitätsklinik Ulm und der Ambulanz der Sonnenberg Klinik gGmbH in Stuttgart eingeschlossen. Die Stichprobenkalkulation erfolgte für die $\mathrm{Ge}$ samtstudie mit dem Hauptzielkriterium Arbeitsfähigkeit (Work Ability Index), mit $G^{\star}$ Power für eine Varianzanalyse mit Messwiederholung (ANOVA). Die Stichprobengröße für die Identifikation einer mittleren Effektstärke $(\mathrm{f}=0,25)$ bei einem Signifikanzniveau von $p<0,05$ und einer Power von 0,95 betrug $n=220$ [30].

Von den insgesamt 367 eingeschlossenen Teilnehmern erhielten wir 205 gültige Werte zur Zufriedenheit mit dem Angebot. In die Regressionsanalyse konnten 189 Teilnehmer eingeschlossen werden. Die endgültige Stichprobe bestand aus $n_{\mathrm{PSIW}}=98$ und $n_{\mathrm{PSOC}}=91($ siehe $\bullet$ Abb. 1$)$.

\section{Variablen}

\section{Zufriedenheit mit dem Angebot aus Nutzerperspektive}

Die unabhängige Variable wurde mit dem Patientenfragebogen zur Erfassung der Zufriedenheit in der Version mit 8 Items (ZUF-8; [32]) erhoben. Er erfasst die globale Patientenzufriedenheit 
Bundesgesundheitsbl 2019 • 62:186-194 https://doi.org/10.1007/s00103-018-2869-x

(c) Der/die Autor(en) 2019

E. Rothermund · R. Kilian · E. M. Balint · E. Rottler · J. von Wietersheim · H. Gündel · M. Hölzer

Wie bewerten Nutzer das neue Versorgungsmodell „Die Psychosomatische Sprechstunde im Betrieb“? Ergebnisse einer kontrollierten Beobachtungsstudie

\section{Zusammenfassung}

Hintergrund. Bei der „Psychosomatischen Sprechstunde im Betrieb" bieten externe Fachpsychotherapeuten einen innerbetrieblichen Erstkontakt an. Im Gegensatz zum bisher etablierten Erstkontakt in der Regelversorgung wird der Arbeitsplatz explizit mit einbezogen und bei Einverständnis des Patienten eine Verzahnung mit innerbetrieblichen Akteuren hergestellt. Das neue Modell eignet sich zur Früherkennung psychischer Störungen und ist effektiv. Verlässliche Daten zur Zufriedenheit der Nutzer fehlten bisher.

Fragestellung. Wie zufrieden sind Nutzer des neuen Versorgungsmodells im Vergleich mit Nutzern der Regelversorgung?

Material und Methoden. 189 Nutzer aus betrieblichen $(n=91)$ und in der
Regelversorgung etablierten $(n=98)$ Sprechstunden machten vor Erstkontakt (t1) und 12 Wochen danach (t2) mittels Selbstbeurteilungsinstrumente Angaben zur Zufriedenheit mit dem Angebot (t2), zur Weiterbehandlung (t2) und zur Depressivität (t1, t2). In einer OLS(Ordinary-Least-Squares)Regressionsanalyse wurde der Einfluss von Behandlungsqualität (klinische Besserung, Weiterbehandlung) und Behandlungsort auf die Nutzerzufriedenheit (abhängige Variable) untersucht, unter Kontrolle der unterschiedlichen Ausgangsdepressivität und des Selektionsbias.

Ergebnisse. Je ausgeprägter die klinische Besserung war, desto zufriedener waren die Nutzer ( $b=1,2, \mathrm{KI} 0,802-1,548)$. Fand eine Anschlussbehandlung statt, war der Mittelwert (MW) der Zufriedenheit um 1,6 Punkte höher (KI 0,236-2,909). Bei Nutzern der betrieblichen Sprechstunde war der MW der Zufriedenheit um 1,8 Punkte höher als in der Regelversorgung (KI 0,322-3,302). Diskussion. Die "Psychosomatische Sprechstunde im Betrieb" wird aus der Perspektive der Nutzer positiv in Bezug auf Behandlungserfolg und Dienstleistungsqualität wahrgenommen.

Schlüsselwörter

Patientenzufriedenheit · Depression . Frühintervention · Reliable Change Index . Arbeitsplatz

\section{How do users assess the new "Psychosomatic Consultation in the Workplace" model of care? Results of a controlled observational trial}

\section{Abstract}

Background. Within the framework of psychosomatic consultation in the workplace (PSIW), external expert psychotherapists offered consultation sessions for employees at the company premises in order to establish a first contact. In contrast to the first contact usually established in regular care, PSIW explicitly focuses on the workplace and enables in-company actors to get closely involved, if the patient's consent is provided. The new model of care helps to identify mental problems at an early stage and is clinically effective. Reliable data considering the user's point of view is missing.

Objectives. How satisfied are users of the new model of care compared to users of regular care?
Material and methods. A total of 189 people from in-company $(n=91)$ or regular medical consultations $(n=98)$ made statements before first contact (t1) and 12 weeks ( 2 ) after the initial contact. They reported satisfaction with the offer ( $\mathrm{t} 2)$, further treatment $(\mathrm{t} 2)$, and depressiveness $(\mathrm{t} 1, \mathrm{t} 2)$ by means of selfassessment tools. On the basis of an OLS (ordinary least squares) regression analysis, we investigated how treatment quality (clinical improvement, further treatment) and the place of treatment influenced user satisfaction (dependent variable), while controlling for selection bias and the different degrees of depression at baseline.

Results. Higher clinical improvement predicted more satisfaction with the received treatment in both $(b=1.2, \mathrm{Cl} 0.802-1.548)$. The mean value (MV) of satisfaction was 1.6 points higher if further treatment took place (CI 0.236-2.909). PSIW users showed a 1.8 points higher MV of satisfaction than people in regular care ( $\mathrm{Cl}$ 0.322-3.302).

Conclusions. PSIW users consider PSIW as positive in terms of treatment success and service quality.

\section{Keywords}

Patient satisfaction - Depression - Early intervention - Reliable change index . Workplace
[33]. Es handelt sich um eine reliable (Cronbachs a 0,90), eindimensionale Skala, die Werte zwischen 8 und 32 annehmen kann [34]. Zusammenhänge zur Einschätzung der Dienstleistungsqualität sind mit einer Sensitivität von $75 \%$ und einer Spezifität von $86 \%$, Zusammenhänge zur Veränderungsmessung mit einer Sensitivität von $59 \%$ und einer Spezifität von $79 \%$ für Stichproben mit Patienten im psychosomatischen Behandlungskontext beschrieben [34]. Für das Instrument wird eine linksschiefe Verteilung berichtet [34]. Das bedeutet, dass damit meist Werte mit einer hohen durchschnittlichen Zufriedenheit bei insgesamt geringer Varianz gemessen werden.

Ursprünglich für den ambulanten Bereich entwickelt [35], wurde die deutsche Version für den stationären Rahmen formuliert [32]. In der vorliegenden Untersuchung erfolgte die Adaption für das Angebot der Psychosomatischen Sprech- stunde. Die entsprechenden Items mit jeweils vier Antwortmöglichkeiten finden sich in $\bullet$ Infobox 2.

\section{Depressivität}

Depressivität wurde anhand der deutschen Übersetzung des Gesundheitsfragebogens für Patienten (Patient Health Questionnaire, PHQ) mit neun Items (PHQ-9) erhoben. Das Ergebnis kann Werte zwischen 0 und 27 annehmen. Der PHQ-9 ist ein valides (Sensitivität 88\%, 
Infobox 2 Formulierung der Items der Zufriedenheitsskala (ZUF-8) in der vorliegenden Untersuchung und der jeweiligen Antwortmöglichkeiten

1. Wie würden Sie die Qualität der Behandlung, die Sie erhalten haben, beurteilen? (ausgezeichnet - gut - weniger gut schlecht)

2. In welchem Maße hat unser Angebot Ihren Bedürfnissen entsprochen? (sie hat fast allen ... - ... den meisten ... - ... nur wenigen ... - ... nicht entsprochen)

3. Würden Sie einem Freund (einer Freundin) die psychosomatische Sprechstunde empfehlen, wenn er oder sie eine ähnliche Hilfe benötigen würde? (eindeutig nicht ich glaube nicht - ich glaube ja - eindeutig ja)

4. Wie zufrieden sind Sie mit dem Ausmaß der Hilfe, welche Sie erhalten haben? (ziemlich unzufrieden - leicht unzufrieden weitgehend zufrieden - sehr zufrieden)

5. Hat die Behandlung, die Sie hier erhielten, Ihnen dabei geholfen, angemessener mit Ihren Problemen umzugehen? (ja, sie half eine ganze Menge - ja, sie half etwas-nein, sie half eigentlich nicht - nein, sie hat mir die Dinge schwerer gemacht)

6. Würden Sie wieder in die psychosomatische Sprechstunde kommen, wenn Sie Hilfe bräuchten? (eindeutig nicht - ich glaube nicht - ich glaube ja - eindeutigja)

7. Wie zufrieden sind Sie mit der Behandlung, die Sie hier erhalten haben, im Großen und Ganzen? (sehr zufrieden - weitgehend zufrieden - leicht unzufrieden - ziemlich unzufrieden)

8. Haben Sie aus heutiger Sicht die Art der Behandlung erhalten, die Sie wollten? (eindeutig nicht - eigentlich nicht - im Allgemeinen ja - eindeutigja)

Spezifität $88 \%$ ) und reliables (Cronbachs a 0,89) Instrument [36]. Entsprechend den diagnostischen Kriterien nach ICD10 können $\mathrm{ab}$ einem Wert von über 10 klinisch relevante Symptome angenommen werden $[36,37]$.

\section{Klinische Veränderung}

Als Maß für die intraindividuelle klinische Veränderung wurde die um den Messfehler korrigierte Differenz zwischen dem Ausgangswert der Depressivität zu t1 und dem Wert der Depressivität 12 Wochen nach Erstkontakt berechnet (t2).

Dazu wurde die Differenz $\mathrm{t} 1$ - t2 zu ihrer eigenen Standardabweichung SA(t1
- t2) ins Verhältnis gesetzt. Das Ergebnis ist der Reliable Change Index [38] für Depressivität $\left(\mathrm{RCI}_{\text {Depr }}\right)$ :

$$
\begin{aligned}
& \mathrm{RCI}_{\text {Depr }}=\frac{\mathrm{t} 1-\mathrm{t} 2}{S A(\mathrm{t} 1-\mathrm{t} 2)} \\
& =\frac{\mathrm{t} 1-\mathrm{t} 2}{S A(\mathrm{t} 2) \sqrt{2\left(1-\alpha_{\mathrm{C}}(\text { PHQ9) })\right.}} .
\end{aligned}
$$

$\alpha_{\mathrm{C}} \quad$ Cronbachs $\alpha$

\section{Psychotherapeutische Weiterbehandlung}

Die Befragten gaben an, ob im Anschluss eine Weiterbehandlung stattgefunden hatte.

Es wurde gefragt, ob es zu einer für den einen Betrieb möglichen/spezifischen Kurzzeitpsychotherapie von 10 Sitzungen oder zur Aufnahme ambulanter Einzel- oder Gruppenpsychotherapie kam bzw. ob eine tagesklinische oder stationäre psychotherapeutische Behandlung begonnen wurde oder ob ein Facharzt für Psychiatrie oder psychosomatische Medizin aufgesucht wurde. Zusätzlich gab es die Möglichkeit, andere Behandlungsformen per Freitext anzugeben. Eine detaillierte Auswertung ergab zu viele Untergruppen, sodass diese Variable dichotomisiert wurde.

\section{Statistische Analysen}

Die Merkmale der Stichprobe werden deskriptiv berichtet. Sie wurden entsprechend dem Variablenniveau als Mittelwert (MW) mit Standardabweichung (SA) oder Anzahl und Prozentwert dargestellt. Mittelwertunterschiede der kontinuierlichen Variablen wurden mittels $T$-Test, Unterschiede in der Verteilung kategorialer oder nominaler Variablen mittels $\mathrm{X}^{2}$-Test überprüft.

Um aufzuklären, ob und inwieweit Behandlungsort und Behandlungserfolg (klinische Besserung, Aufnahme weiterer Psychotherapie) die Zufriedenheit von Nutzern eines medizinischen Angebotes beeinflussen, wurde eine OLS(Ordinary-Least-Squares-)-Regression mit einer Propensity-Score-Adjustierung zur Kontrolle des Selektionsbias [39] durchgeführt. Die Schätzung der Propensity-Scores erfolgte auf der Basis eines logistischen Regressionsmodells mit den unabhängigen Variablen: Alter, Geschlecht, Familienstand, Umfang der Erwerbstätigkeit, berufliche Qualifikation, Vorbehandlung, Symptomdauer und vorangegangenes Inanspruchnahmeverhalten.

Die abhängige Variable des multiplen linearen Regressionsmodells war "Zufriedenheit mit dem Angebot“. Die unabhängigen Variablen waren „klinische Besserung“, „Aufnahme weiterer Psychotherapie“, „Behandlungsort“ sowie „Depressivität vor Erstkontakt“ und „Propensity-Score“. Zusätzlich wurden folgende Interaktionseffekte geprüft: Erkrankungsschwere (Depressivität vor Erstkontakt)*Behandlungserfolg und Behandlungsort ${ }^{\star}$ Behandlungserfolg. Zur leichteren Interpretation der Regressionskoeffizienten wurde der Mittelwert der Depressivität zentriert.

Das Signifikanzniveau wurde auf $p=0,05$ festgelegt. Es werden die unstandardisierten Regressionskoeffizienten $(b)$ mit 95\% korrigiertem Konfidenzintervall, die standardisierten Regressionskoeffizienten, das Signifikanzniveau sowie die Gütekriterien des Modells dargestellt. Die abhängige Variable weist eine linksschiefe Verteilung auf. Deshalb erfolgte die Schätzung der Standardfehler der Regressionskoeffizienten mittels nonparametrischem Bootstrapping mit 1000 Replikationen. Alle Analysen wurden mittels SPSS Version 25 durchgeführt.

\section{Ergebnisse}

\section{Deskriptive Ergebnisse}

Die Daten von 189 Teilnehmern im Betrieb $\left(n_{\mathrm{PSIW}}=98\right)$ und in der Regelversorgung $\left(n_{\mathrm{PSOC}}=91\right)$ wurden analysiert. - Tab. 1 gibt einen Überblick über die Daten. Die Teilnehmer waren durchschnittlich 44,6 $\pm 10,86$ Jahre alt und zu 55,6\% männlich. Die Zufriedenheit lag in der Gesamtstichprobe bei einem Wert von 25,4 $\pm 4,88$ Punkten auf einer Skala von 8-32. Im Mittel betrug der Wert für Depressivität im PHQ-9 vor dem Erstkontakt 12,7 \pm 6,22 Punkte, nach 12 Wochen 9,6 \pm ,18 Punkte auf einer Skala von 0-27 Punkten. Insgesamt befanden sich mit 50,8\% etwa die Hälfte der Teilnehmer seit dem Erst- 


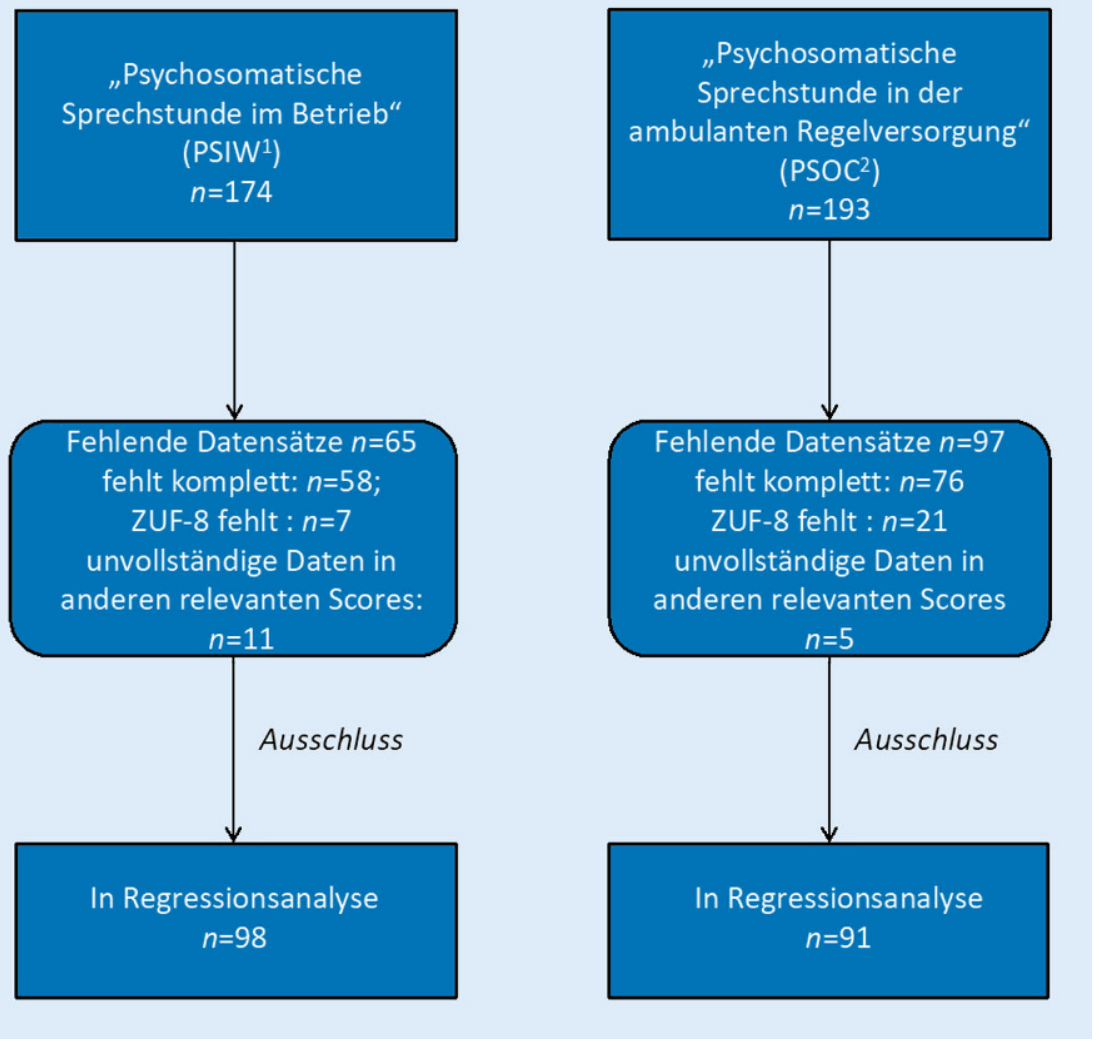

Abb. 1 A Rekrutierungsverlauf und Einschluss von Teilnehmern in die Analyse sowie Anzahl ausgeschlossener Teilnehmer unter Angabe der Gründe. 'PSIW Psychosomatic Consultation in the Workplace, ${ }^{2}$ PSOC Psychosomatic Outpatient Care, $n$ Anzahl, ZUF-8 Fragebogen zur Messung der Patientenzufriedenheit

kontakt in weiterer Behandlung. Die Teilnehmenden aus der Betriebssprechstunde waren im Gegensatz zu denen der Regelversorgung signifikant älter, häufiger männlich, weniger depressiv und seltener in weiterer Behandlung.

Zur Analyse der Non-Responder (siehe Abb. 1) wurde eine logistische Regression mit "Teilnahme" als dichotomer, abhängiger Variable durchgeführt. Die Ergebnisse der fehlenden im Vergleich zu den eingeschlossenen Teilnehmern zeigte, dass zunehmendes Alter mit einer geringfügig größeren Wahrscheinlichkeit teilzunehmen einherging (OR 0,01, KI 0,005-0,014). Kein Einfluss auf das Antwortverhalten konnte in Bezug auf das Geschlecht, die Erkrankungsschwere bei Erstkontakt oder den Behandlungsort in der Stichprobe nachgewiesen werden.

\section{Ergebnisse der multiplen linearen Regression}

Die Ergebnisse der multiplen linearen Regression (siehe $\bullet$ Tab. 2) zeigen, dass die Zufriedenheit der Nutzer vor allem durch das klinische Behandlungsergebnis beeinflusst wurde. Für beide Behandlungsorte (Betriebssprechstunde, übliche ambulante Versorgung) galt: Je ausgeprägter die klinische Besserung war, desto zufriedener äußerten sich die Nutzer $(b=1,2$, KI 0,802 bis $1,548, p=0,001)$. Fand eine Anschlussbehandlung über die zwei Sitzungen der Sprechstunde hinaus statt, war der MW der Zufriedenheit der Nutzer beider Behandlungsorte um 1,6 höher $(b=1,6$, KI 0,236-2,909, $p=0,021)$, als wenn dies nicht der Fall war. Der Mittelwert der Zufriedenheit mit dem Angebot war bei den Nutzern der Sprechstunde im Betrieb um 1,8 Punkte höher als in der Regelversorgung ( $b=1,8, \mathrm{KI}$ $0,322-3,302, p=0,024)$.
Die Prüfung der Interaktionseffekte zeigt keine signifikanten Ergebnisse.

Durch die in das Modell einbezogenen Variablen konnte laut $R^{2}$ eine Varianz von $24,0 \%$ aufgeklärt werden $\left(R^{2}\right.$ korrigiert 0,219$)$. Der Vergleich der standardisierten Regressionskoeffizienten zeigt den stärksten Einfluss im Modell für die Variable „klinische Veränderung“.

\section{Diskussion}

In einer prospektiven kontrollierten Studie mit 189 Teilnehmern, davon $n_{\text {PSIW }}=98$ in einer ,Psychosomatischen Sprechstunde im Betrieb" und $n_{\mathrm{PSOC}}=91$ in der Regelversorgung, wurde untersucht, welchen Einfluss Behandlungserfolg und Behandlungsort (Betrieb oder Regelversorgung) 12 Wochen nach Erstkontakt auf die Zufriedenheit der Nutzer hatte. Dabei zeigte sich, dass das Ausmaß der klinischen Änderung den größten Anteil an der Aufklärung der Varianz hatte. Einen bedeutsamen Einfluss besaß auch der Behandlungsort. Die Nutzer des betrieblichen Angebots waren um 1,8 Punkte zufriedener als die Nutzer der Regelversorgung.

\section{Behandlungserfolg und Zufriedenheit}

Je ausgeprägter sich die Depressivität als Maß für die klinische Beeinträchtigung zwischen Erstkontakt und Nachbefragung besserte, desto zufriedener äußerten sich die Nutzer. Auch eine Weiterbehandlung über zwei Sitzungen hinaus verbesserte die Zufriedenheit. Damit relativieren die vorliegenden Ergebnisse die These, dass Menschen im Allgemeinen gemäß dem Prinzip der sozialen Erwünschtheit antworten, ihrer Dankbarkeit Ausdruck verleihen möchten oder, um kognitive Dissonanz zu minimieren, dazu neigen, das eigene Tun bzw. Mitwirken positiv zu bewerten [40]. Auch widersprechen die Befunde der von Crow und Kollegen in einem systematischen Review 2002 [27] geäußerten Kritik, dass hohe Werte für Zufriedenheit vorlägen, auch wenn nachweislich weiter Gesundheitsprobleme bestünden, da Menschen „zufrieden“ im Sinne von „ausreichend“ interpretierten. 


\begin{tabular}{|c|c|c|c|c|}
\hline Merkmal & $\begin{array}{l}\text { Gesamte Stichpro- } \\
\text { be }(n=189) \\
\%(n) / M W \pm \text { SA }\end{array}$ & $\begin{array}{l}\text { Psychosomatische Sprechstun- } \\
\text { de im Betrieb (PSIW) }(n=98) \\
\%(n) / M W \pm \text { SA }\end{array}$ & $\begin{array}{l}\text { Psychosomatische Sprechstunde in } \\
\text { der Regelversorgung (PSOC) }(n=91) \\
\%(n) / M W \pm \text { SA }\end{array}$ & \\
\hline Alter & $44,6 \pm 10,86$ & $47,1 \pm 9,55$ & $42,0 \pm 11,60$ & $p=0,001^{\mathrm{a}}$ \\
\hline Geschlecht (männlich) & $55,6(105)$ & $68,4(67)$ & $41,8(38)$ & $p<0,000^{b}$ \\
\hline Zufriedenheit mit dem Angebot & $25,4 \pm 4,88$ & $26,5 \pm 4,50$ & $24,2 \pm 5,001$ & $p=0,001^{\mathrm{a}}$ \\
\hline $\begin{array}{l}\text { Depressivität vor Erstkontakt } \\
(\mathrm{PHQ}-9, \mathrm{t} 1)\end{array}$ & $12,7 \pm 6,22$ & $11,0 \pm 5,76$ & $14,3 \pm 6,23$ & $p<0,000^{\mathrm{a}}$ \\
\hline $\begin{array}{l}\text { Depression } 12 \text { Wochen nach } \\
\text { Erstkontakt (PHQ-9, t2) }\end{array}$ & $9,6 \pm 6,18$ & $8,5 \pm 6,03$ & $10,9 \pm 6,13$ & $p=0,006^{\mathrm{a}}$ \\
\hline $\begin{array}{l}\text { Weitere Behandlung in An- } \\
\text { spruch genommen (ja) }\end{array}$ & $50,8(96)$ & $38,8(38)$ & $63,7(58)$ & $p=0,001^{b}$ \\
\hline $\begin{array}{l}{ }^{\mathrm{a}} T \text {-Test } \\
{ }^{\mathrm{b}} \mathrm{X}^{2} \text {-Test }\end{array}$ & & & & \\
\hline
\end{tabular}

\begin{tabular}{|c|c|c|}
\hline Eingeschlossene Variablen & $\begin{array}{l}\text { Unstandardisierter Regres- } \\
\text { sionskoeffizient } b(95 \% \\
\mathrm{KI})^{\mathrm{a}}\end{array}$ & $\begin{array}{l}\text { Standardisierter } \\
\text { Regressionskoeffi- } \\
\text { zient }\end{array}$ \\
\hline $\begin{array}{l}\text { Klinische Veränderung/ } \\
\text { Behandlungserfolg seit Erstgespräch }\end{array}$ & $1,182(0,802-1,548)^{* * *}$ & 0,459 \\
\hline $\begin{array}{l}\text { Weiterbehandlung über } 2 \text { Sitzungen } \\
\text { hinaus }\end{array}$ & $1,586(0,236-2,909)^{*}$ & 0,163 \\
\hline $\begin{array}{l}\text { Behandlungsort Psychosomatische } \\
\text { Sprechstunde (im Betrieb oder in der } \\
\text { ambulanten üblichen Versorgung) }\end{array}$ & $1,844(0,322-3,302)^{*}$ & 0,193 \\
\hline $\begin{array}{l}\text { Depressivität vor Erstgespräch (Mittel- } \\
\text { wert zentriert) }\end{array}$ & $-0,195(0,341 \text { bis }-0,049)^{* *}$ & $-0,248$ \\
\hline \multirow[t]{2}{*}{ Propensity-Score } & $1,178(-1,096$ bis 3,730$)$ & 0,085 \\
\hline & \multicolumn{2}{|c|}{$\begin{array}{l}\text { Regressionskonstante: } 21,787, \mathrm{R}^{2}: 0,240, \text { Fallzahl } \\
n=189\end{array}$} \\
\hline \multicolumn{3}{|c|}{$\begin{array}{l}{ }^{*} p \leq 0,05,{ }^{* *} p \leq 0,01,{ }^{* *} p \leq 0,001 \\
{ }^{*} \text { nonparametrisches Bootstrapping mit } 1000 \text { Replikationen }\end{array}$} \\
\hline
\end{tabular}

Der Befund der vorliegenden Arbeit steht im Gegensatz zu einer Studie, die, zwar mit anderen Instrumenten, in einem ambulanten Angebot $\mathrm{zu}$ dem Schluss kam, dass die Zufriedenheit vorwiegend von patientenseitigen Faktoren bestimmt wird [41]. Der Befund einer allgemein hohen Zufriedenheit wurde in der vorliegenden Stichprobe repliziert. Die Ergebnisse stehen im Einklang mit Daten einer Erhebung in Deutschland an 50.000 Patienten, die ebenfalls mittels ZUF-8 befragt wurden und Zusammenhänge zwischen Zufriedenheit und Therapieerfolg belegen [34]. Etliche Studien in der internationalen Literatur über verschiedene Populationen, Messinstrumente und Kulturen hinweg bestätigen ebenfalls den Zusammenhang zwischen Behandlungserfolg und Zufriedenheit [27]. Eine Stärke der vorliegenden Untersuchung ist, dass der Behandlungserfolg nicht nur über zwei Parameter (geglückte Weiterbehandlung und klinische Veränderung) gemessen wird, sondern dass die klinische Veränderungsmessung durch die Anwendung des Reliable Change Index um den Messfehler bereinigt ist. Somit können bedeutungsvolle Änderungen angenommen werden.

\section{Dienstleistungsqualität und Zufriedenheit}

Das zweite zentrale Ergebnis der vorliegenden Untersuchung ist, dass die Nutzer des betrieblichen Angebots um 1,8 Punkte zufriedener als die Nutzer der Regelversorgung waren. Das Merkmal Behandlungsort Betrieb umfasst, wie in der Einleitung beschrieben, eine ört- liche und inhaltlich maßgeschneiderte Anpassung der bisherigen Gesundheitsleistung Psychosomatische Sprechstunde [20, 22, 31]. Der Behandler sucht den Betroffenen im Betrieb auf. Inhaltlich wird der Arbeitsplatz sowohl als Stressor als auch als Ressource explizit mit einbezogen. Obwohl der Arbeitsplatzbezug in der Sozialanamnese bei jedem psychosomatisch-psychiatrisch-psychotherapeutischen Erstkontakt Eingang finden könnte, wird dies von Therapeuten und Ärzten meist wenig ausreichend thematisiert [42]. Oft fehlt der direkte Kontakt zu innerbetrieblichen Akteuren [18] und/oder detailliertes Wissen zu Inhalten und Abläufen am jeweiligen Arbeitsplatz [42, 43]. Aus diesem Grund kann davon ausgegangen werden, dass die größere Zufriedenheit der Nutzer im Betrieb der maßgeschneiderten Dienstleistungsqualität geschuldet ist. Dass dies Teil des mit dem ZUF-8 gemessenen Konstrukts ist, haben Kriz und Kollegen in der bereits oben genannten Stichprobe eindrucksvoll belegt [34].

Nicht ausschließen lässt sich, dass die höhere Zufriedenheit in der betrieblichen Sprechstunde über eine geringere Depressivität vermittelt wird. Wie bereits in Voruntersuchungen der Stichprobe beschrieben, werden Menschen mit psychischen Problemen im Betrieb früher erreicht und haben bei Erstkontakt geringer ausgeprägte Beschwerden als Menschen, die eine ambulante psychosomatische Sprechstunde in Klinikräumen aufsuchen $[16,17,23]$. Um dieser Verzerrung der Ergebnisse zu begegnen, wurde das Modell für die Depressivität vor Erst- 
kontakt kontrolliert. Der Effekt konnte unverändert beobachtet werden.

Für die Einschätzung der Durchführbarkeit einer "Psychosomatischen Sprechstunde im Betrieb“ ist der vorliegende Befund damit ein wichtiger Beitrag. Die allerersten Publikationen zum Thema betriebsnaher, integrierter Versorgungsleistungen berichteten entweder von persönlichen Eindrücken, dass das Angebot Akzeptanz findet [44], schlossen aus Nutzerverhalten indirekt auf Akzeptanz [45] oder untersuchten zwar die Akzeptanz mittels validierten Instruments, konnten die Ergebnisse jedoch nicht mit einer Kontrollgruppe vergleichen [46].

\section{Patientenzufriedenheit als Maß für die Nutzerperspektive}

Obwohl sich das Konstrukt Patientenzufriedenheit weitgehend etabliert hat [26-29], wird kontrovers diskutiert [47], ob das, was gemessen wird, mit dem Angebot zu tun hat oder vorwiegend von persönlichen Merkmalen beeinflusst wird [41, 47]. Strittig ist auch, ob die Patientenzufriedenheit beeinflusst wird von der Art der Erhebung [29] und den Konsequenzen daraus, z.B. dem einseitigen Einsatz als Benchmarking [48].

Die Bedeutung, die der Einschätzung der Zufriedenheit der Nutzer mit dem neuen Versorgungsmodell PSIW beikommt, verdeutlichen folgende Argumente:

Erstens ist die Patienten- oder Nutzerzufriedenheit ein verlässlicher Hinweis, ob ein Behandlungsangebot für einen Behandlungssuchenden verfügbar, leicht erreichbar und erschwinglich ist, eine ausreichende Behandlungsqualität aufweist und vom Hilfesuchenden akzeptiert wird [28].

Zweitens hat die Akzeptanz durch die Hilfesuchenden zentrale Bedeutung für die Implementierung eines Angebotes [49].

Drittens ist, ähnlich wie die Akzep$\tan z$ durch die Nutzer, deren subjektive Bewertung über die Messung klinischer Veränderung hinaus entscheidend. Entsprechend der Konzeptualisierung von Patienten-/Nutzerzufriedenheit nach
Ware et al. [50] kann davon ausgegangen werden, dass Unterschiede in der Zufriedenheit die Realität der Versorgung $\mathrm{zu}$ einem Teil wiedergeben, wenngleich sie auch die persönlichen Vorlieben und Erwartungen des Nutzers widerspiegeln [50]. So lag mit der Messung der Zufriedenheit eine Bewertung bezüglich Sinn und Nutzen des neuen Versorgungsangebotes aus der Nutzerperspektive vor.

Viertens ist die Nutzerperspektive an der Schnittstelle zwischen Arbeit und seelischer Gesundheit essenziell, da psychische Erkrankungen häufig mit einer Angst vor Stigmatisierung verbunden sind [12].

Ein weiterer Kritikpunkt der Forschung im Bereich Nutzer-/Patientenzufriedenheit sind die mangelnde $\mathrm{Zu}$ verlässigkeit/Reliabilität und Validität der zahlreichen Instrumente. Aus diesem Grund wurde der ZUF-8 gewählt, der sowohl für den deutschsprachigen Raum [32, 33] als auch an einer großen Stichprobe psychosomatischer Patienten bereits sorgfältig validiert wurde [34].

Einschränkend muss zudem gesagt werden, dass die vorliegenden Ergebnisse nicht generalisierbar sind, da sie in nur einer Region Deutschlands und nicht unter randomisierten Bedingungen durchgeführt werden konnten. Aus der Perspektive der Versorgungsforschung ist jedoch die Analyse unter Routinebedingungen eine besondere Stärke des hier gewählten Ansatzes.

\section{Fazit}

Die Anforderungen einer modernen Arbeitswelt liegen unter anderem in der Verdichtung von Arbeitsprozessen, elektronischer Kommunikation, der Notwendigkeit zu räumlicher Flexibilität und der Fähigkeit und Bereitschaft zu lebenslanger beruflicher Veränderung. Diese Anforderungen können psychische Beschwerden auslösen oder verstärken [2-4]. Historisch gesehen bestand die Reaktion auf Missstände in der Arbeitswelt häufig in Maßnahmen zur Förderung der individuellen Gesundheit der Beschäftigten [25], was in Deutschland z.B. von den Gewerkschaften mit dem Hinweis auf Änderungen an un- günstigen Arbeitsbedingungen kritisch diskutiert wird [24].

Auf der anderen Seite kann ein Individuum mit psychischer Belastung oder Beschwerden in der Arbeitswelt zunehmend schwerer bestehen bzw. meist nur mit einem erhöhten individuellen Aufwand. Das liegt daran, dass unsere Arbeitswelt meist in einem hohen Maß soziale und kommunikative Fähigkeiten wie auch Fähigkeiten der Selbstregulation erfordert, die bei psychischen und psychosomatischen Erkrankungen häufig begrenzt sind, wodurch die Teilhabe am Arbeitsleben eingeschränkt wird.

Aus diesem Grund sollte Arbeit als soziale Determinante psychischer Gesundheit begriffen und in den Behandlungsprozess integriert werden [42]. Dies ist aus Sicht der Autoren über eine stärkere Vernetzung mit betrieblichen Akteuren wie in dem Modell der "Psychosomatischen Sprechstunde im Betrieb“ möglich.

Aus der Perspektive der Nutzer aus drei teilnehmenden Großbetrieben wurde die „Psychosomatische Sprechstunde im Betrieb" sowohl positiv in Bezug auf den Behandlungserfolg (bestehend aus klinischer Veränderung und Vermittlung einer Weiterbehandlung) als auch auf die Qualität dieser Dienstleistung wahrgenommen. Über das Potenzial von Früherkennung und Effektivität aus klinischer Sicht hinaus wird das untersuchte Modell von der Nutzergruppe selbst insgesamt als passend akzeptiert.

\section{Korrespondenzadresse}

\section{Dr. Eva Rothermund}

Klinik für Psychosomatische Medizin und Psychotherapie, Universitätsklinikum Ulm Alberteinstein-Allee 23, $89081 \mathrm{Ulm}$,

Deutschland

eva.rothermund@uni-ulm.de

Danksagung. Wir danken allen teilnehmenden Patienten, Anita Gutbrod, Petra Beschoner und den kooperierenden Betriebs- und Werksärzten für ihre Unterstützung sowie Gabriele Kreutzer-Stierle und Firuzan Kundt für das Datenmanagement. Besonderer Dank gilt Cornelia Mayer für die sprachliche Unterstützung.

Förderung. Die Studie wurde durch die Nachwuchsakademie Versorgungsforschung Baden-Württemberg des Ministeriums für Wissenschaft, Forschung und Kunst in Kooperation mit dem Ministerium für Arbeit, Soziales, Familie, Frauen und ältere Menschen Baden-Württemberg gefördert. Die Firma CASSIDIAN 
unterstützte die Evaluierung der "Psychosomatischen Sprechstunde im Betrieb“ im Rahmen ihres Betrieblichen Gesundheitsmanagements.

\section{Einhaltung ethischer Richtlinien}

Interessenkonflikt. E. Rothermund und E.M. Balint arbeiteten als Assistenz- und Fachärztinnen in beiden Ambulanzen. J. von Wietersheim, H. Gündel und M. Hölzer leiten Kliniken bzw. Betriebsambulanzen, die Kooperationsverträge mit Betrieben für die „Psychosomatische Sprechstunde im Betrieb" vereinbart haben. E. Rothermund, E.M. Balint, J. von Wietersheim, H. Gündel und M. Hölzer haben gegen Honorar in interessierten Unternehmen Vorträge oder Workshops zum Thema gehalten. E. Rottler und R. Kilian geben an, dass kein Interessenkonflikt besteht.

Die vorliegende Studie wurde im Deutschen Register klinischer Studien unter der Nummer DRKS00003184 registriert. Die Studie wurde unter Zustimmung der Ethikkommission der Universität Ulm (No224/11), im Einklang mit nationalem Recht sowie gemäß der Deklaration von Helsinki von 1975 (in der aktuellen, überarbeiteten Fassung) durchgeführt. Von allen beteiligten Patienten liegt eine Einverständniserklärung vor.

Open Access. Dieser Artikel wird unter der Creative Commons Namensnennung 4.0 International Lizenz (http://creativecommons.org/licenses/by/4.0/deed. de) veröffentlicht, welche die Nutzung, Vervielfältigung, Bearbeitung, Verbreitung und Wiedergabe in jeglichem Medium und Format erlaubt, sofern Sie den/die ursprünglichen Autor(en) und die Quelle ordnungsgemäßnennen, einen Link zur Creative Commons Lizenz beifügen und angeben, ob Änderungen vorgenommen wurden.

\section{Literatur}

1. Jacobi F, Höfler M, Siegert J et al (2014) Twelvemonth prevalence, comorbidity and correlates of mental disorders in Germany: The Mental Health Module of the German Health Interview and Examination Survey for Adults (DEGS1-MH). Int J Methods Psychiatr Res. https://doi.org/10.1002/ mpr.1439

2. Theorell T, Hammarstrom A, Aronsson Get al (2015) A systematic review including meta-analysis of workenvironment and depressive symptoms. BMC Public Health 15:738. https://doi.org/10.1186/ s12889-015-1954-4

3. Health and Safety Executive (HSE) (2017) Workrelated stress, depression or anxiety statistics in Great Britain 2017

4. de Vries H, Fishta A, Weikert B et al (2017) Determinants of sickness absence and return to work among employees with common mental disorders: a scoping review. J Occup Rehabil. https://doi.org/10.1007/s10926-017-9730-1

5. Thornicroft G, Chatterji S, Evans-Lacko $S$ et al (2017) Undertreatment of people with major depressive disorder in 21 countries. Br J Psychiatry 210:119-124. https://doi.org/10.1192/bjp.bp.116. 188078

6. Wang Y, Henriksen CA, ten Have M et al (2017) Common mental disorder diagnosis and need for treatment are not the same: findings from the NEMESIS Study. Adm Policy Ment Health Ment
Health Serv Res 44:572-581. https://doi.org/10. 1007/s10488-016-0745-2

7. Whiteford HA, Harris MG, McKeon G et al (2013) Estimating remission from untreated major depression: a systematic review and meta-analysis. Psychol Med 43:1569-1585. https://doi.org/10. 1017/S0033291712001717

8. Wang PS, Angermeyer M, Borges G et al (2007) Delay and failure in treatment seeking after first onset of mental disorders in the World Health Organization's World Mental Health Survey Initiative. World Psychiatry 6:177-185

9. OECD (2015) Fit mind, fit job: from evidence to practice in mental health and work. Mental health and work. OECD, Paris https://doi.org/10.1787/ 9789264228283-en

10. Hung Cl, Liu CY, Yang CH (2017) Untreated duration predicted the severity of depression at the twoyear follow-up point. PLoS ONE 12:1-11. https:// doi.org/10.1371/journal.pone.0185119

11. Oluboka OJ, Katzman MA, Habert J et al (2018) Functional recovery in major depressive disorder: providing early optimal treatment for the individual patient. Int J Neuropsychopharmacol 21:128-144. https://doi.org/10.1093/ijnp/pyx081

12. Clement S,Schauman O,Graham Tetal (2015) What is the impact of mental health-related stigma on help-seeking? A systematic review of quantitative and qualitative studies. Psychol Med 45:11-27. https://doi.org/10.1017/S0033291714000129

13. Schomerus G, Stolzenburg S, Freitag S et al (2018) Stigma as a barrier to recognizing personal mental illness and seeking help: a prospective study among untreated persons with mental illness. Eur Arch Psychiatry Clin Neurosci. https://doi.org/10. 1007/s00406-018-0896-0

14. Magaard JL, Seeralan T, Schulz Hetal (2017) Factors associated with help-seeking behaviour among individuals with major depression: a systematic review. PLoS ONE 12:e176730. https://doi.org/10 1371/journal.pone.0176730

15. Stegmann R, Schröder U (2018) Anders Gesund - Psychische Krisen in der Arbeitswelt, 1. Aufl. https://doi.org/10.1007/978-3-658-17882-6

16. Rothermund E, Kilian R, Rottler E et al (2017) Improving access to mental health care by delivering psychotherapeutic care in the workplace: a crosssectional exploratory trial. PLOS ONE 12:e169559. https://doi.org/10.1371/journal.pone.0169559

17. Barrech A, Kilian R, Rottler Eet al (2018) Do working conditions of patients in psychotherapeutic consultation in the workplace differ from those in outpatient care? Results from an observational study. Int J Environ Res Public Health 15:227. https://doi.org/10.3390/ijerph15020227

18. Rothermund $E$, Michaelis $M$, Jarczok $M N$ et al (2018) Prevention of common mental disorders in employees. Perspectives on collaboration from three health care professions. Int J Environ Res Public Health 15:1-13. https://doi.org/10.3390/ ijerph 15020278

19. Martin A, Fisher CD (2014) Understanding and improving managers' responses to employee depression. Ind Organ Psychol 7:270-274. https:// doi.org/10.1111/iops.12147

20. BalintEM, von Wietersheim J, Gündel Het al (2018) Die psychosomatische Sprechstunde im Betrieb - ein Zukunftsmodell? Prax Klin Verhaltensmed Rehabil 101:38-47

21. Thomä H, Kächele H (2006) Psychoanalytische Therapie.Grundlagen, 3. Aufl. Springer, Heidelberg

22. Rothermund E, Hölzer M, Wegewitz U (2018) Die psychosomatische Sprechstunde im Betrieb -
Angebot mit Konsiliarcharakter Psychosomatische Sprechstunde im. Psychother Dialog 19:50-54

23. Rothermund $E$, Gündel $H$, Rottler $E$ et al (2016) Effectiveness of psychotherapeutic consultation in the workplace: a controlled observational trial. BMC Public Health 16:891. https://doi.org/10. 1186/s12889-016-3567-y

24. Deutscher Gewerkschaftsbund Bundesvorstand (2014) Stellungnahme des Deutschen Gewerkschaftsbundes (DGB) zur Verbändeanhörung des Bundesministeriums für Gesundheit zum Referentenentwurf: „Entwurf eines Gesetzes zur Stärkung der Gesundheitsförderung und Prävention (Präventionsgesetz - PrävG)“. DGB Bundesvorstand VB Annelie Buntenbach, Abteilung Sozialpolitik, Berlin

25. Michie S (2002) Causes and management of stress at work. Occup Environ Med 59:67-72. https://doi. org/10.1136/oem.59.1.67

26. Lecher S, Satzinger W, Trojan A, Koch U (2002) Patientenorientierung durch Patientenbefragungen als ein Qualitätsmerkmal der Krankenversorgung. Bundesgesundheitsblatt Gesundheitsforschung Gesundheitsschutz 45:3-12. https://doi.org/10. 1007/s103-002-8379-8

27. Crow R, Gage H, Hampson S et al (2002) The measurement of satisfaction with healthcare: implications for practice from a systematic review of the literature. Health Technol Assess 6:1-244. https://doi.org/10.3310/hta6320

28. Mpinga EK, Chastonay P (2011) Satisfaction of patients: a right to health indicator? Health Policy (New York) 100:144-150. https://doi.org/10.1016/ j.healthpol.2010.11.001

29. Hawthorne G (2006) Review of patient satisfaction measures. Australian Government Department of Health and Ageing, Canberra

30. Rothermund E, Kilian R, Hoelzer M et al (2012) „Psychosomatic consultation in the workplace" - a new model of care at the interface of company-supported mental health care and consultation-liaison psychosomatics: design of a mixed methods implementation study. BMC Public Health 12:780. https://doi.org/10.1186/ 1471-2458-12-780

31. Rothermund E, Gündel H, Kilian R et al (2014) Behandlung psychosomatischer Beschwerden im Arbeitskontext - Konzept und erste Daten. Z Psychosom Med Psychother 60:177-189 (Treatment of psychosomatic disorders occurring in the vocational context - concept and first findings)

32. Schmidt J, Lamprecht F, Wittmann W (1989) Satisfaction with inpatient care development of a questionnaire and first validity assessments. Psychother Med Psychol (Stuttg) 39:248-255 (Zufriedenheit mit der stationärenVersorgung. Entwicklung eines Fragebogens und erste Validitätsuntersuchungen)

33. Schmidt J, Wittmann WW (2002) ZUF_8. Fragebogen zur Messung der Patientenzufriedenheit In: Brähler E, Schumacher J, Strauß B (Hrsg) Diagnostische Verfahren in der Psychotherapie. Hogrefe, Göttingen, S392-396

34. Kriz D, Nuebling R, Steffanowski A et al (2008) Patientenzufriedenheit in der stationaeren Rehabilitation: Psychometrische Reanalyse des ZUF8 auf der Basis multizentrischer Stichproben verschiedener Indikation. ZMed Psychol 17:67-79

35. Larsen DL, Attkisson CC, Hargreaves WA, Nguyen TD (1979) Assessment of client/patient satisfaction: development of a general scale. Eval Program Plann 2:197-207. https://doi.org/10.1016/01497189(79)90094-6 


\section{Leitthema}

36. Kroenke K, Spitzer RL, Williams JBW, Löwe B (2010) The Patient Health QuestionnaireSomatic, Anxiety, and Depressive Symptom Scales: a systematic review. Gen Hosp Psychiatry 32:345-359

37. Kroenke K, Spitzer RL, Williams JBW (2001) The PHQ-9: validity of a brief depression severity measure. J Gen Intern Med 16:606-613

38. Jacobson N, Truax P (1991) Clinical significance: a statistical approach to defining meaningful change in psychotherapy research. J Consult Clin Psychol 59:12-19. https://doi.org/10.1037/0022006X.59.1.12

39. Austin PC (2011) An introduction to propensity score methods for reducing the effects of confounding in observational studies. Multivariate Behav Res 46:399-424. https://doi.org/10.1080/ 00273171.2011 .568786

40. LeVois M, Nguyen TD, Attkisson CC (1981) Artifact in client satisfaction assessment. Experience in community mental health settings. Eval Program Plann 4:139-150. https://doi.org/10.1016/01497189(81)90004-5

41. Pauselli L, Galletti C, Verdolini N et al (2017) Predictors of client satisfaction with outpatient mental health clinic services in Italy and New York. Community Ment Health J 54:1-9. https://doi.org/ 10.1007/s10597-017-0196-6

42. Alsdorf N, Engelbach U, Flick S et al (2017) Psychische Erkrankungen in der Arbeitswelt. Analysen und Ansätze zur therapeutischen und betrieblichen Bewältigung. transcript, Bielefeld

43. Bethge M (2017) Rehabilitation und Teilhabe am Arbeitsleben. Bundesgesundheitsblatt Gesundheitsforschung Gesundheitsschutz 60:427-435. https://doi.org/10.1007/s00103-017-2519-8

44. Wege N, Janssen B, Rothermund E (2015) Betriebsnahe Versorgungsnetzwerke - Beispiele guter Praxis in Deutschland. In: Angerer $\mathrm{P}$, Glaser J, Gündel H et al (Hrsg) Psychische und psychosomatische Gesundheit in der Arbeit, 1. Aufl. ecomed Medizin, Heidelberg, München, Landsberg, Frechen, Hamburg, S 299-308

45. Bode K, Wunsch E-M, Finger F, Kröger C (2016) Interdisciplinary care of employees with mental disorders: a fact check using the Salzgittermodel as an example. Psychother Psychosom Med Psychol 66:235-241 (Interdisziplinäre Versorgung von Arbeitnehmern mit psychischen Störungen: Ein Faktencheck am Beispiel des SalzgitterModells)

46. Hölzer M (2012) Psychische Gesundheit im Betrieb. Psychother Dialog 13:52-55

47. Gill L, White $L$ (2009) A critical review of patient satisfaction. Leadersh Health Serv 22:8-19. https:// doi.org/10.1108/17511870910927994

48. Hekkert KD, Cihangir S, Kleefstra SM et al (2009) Patient satisfaction revisited: a multilevel approach. Soc Sci Med 69:68-75. https://doi.org/ 10.1016/j.socscimed.2009.04.016

49. Damschroder LJ, Aron DC, Keith RE et al (2009) Fostering implementation of health services research findings into practice: a consolidated framework for advancing implementation science. Implement Sci 4:50. https://doi.org/10.1186/ 1748-5908-4-50

50. Ware JE, Snyder MK, Wright WR, Davies AR (1983) Defining and measuring patient satisfaction with medical care. Eval Program Plann 6:247-263. https://doi.org/10.1016/0149-7189(83)90005-8 